\title{
The expanding dusty bipolar nebula around the nova V1280 Scorpi ${ }^{\star}$
}

\author{
O. Chesneau ${ }^{1}$, E. Lagadec $^{2}$, M. Otulakowska-Hypka ${ }^{3}$, D. P. K. Banerjee ${ }^{4}$, C. E. Woodward ${ }^{5}$, E. Harvey ${ }^{1}$, A. Spang ${ }^{1}$, \\ P. Kervella ${ }^{6}$, F. Millour ${ }^{1}$, N. Nardetto ${ }^{1}$, N. M. Ashok ${ }^{4}$, M. J. Barlow ${ }^{7}$, M. Bode ${ }^{8}$, A. Evans ${ }^{9}$, D. K. Lynch ${ }^{10}$, \\ T. J. O’Brien ${ }^{11}$, R. J. Rudy ${ }^{10}$, and R. W. Russell ${ }^{10}$
}

${ }^{1}$ Laboratoire Lagrange, UMR 7293, Univ. Nice Sophia-Antipolis, CNRS, Observatoire de la Côte d'Azur, 06300 Nice, France e-mail: Olivier.Chesneau@oca.eu

2 European Southern Observatory, Karl-Schwarzschild-Strasse 2, 85748 Garching bei München, Germany

${ }^{3}$ N. Copernicus Astronomical Center, Polish Academy of Sciences, Bartycka 18, 00-716 Warsaw, Poland

${ }^{4}$ Physical Research Laboratory, Navrangpura, Ahmedabad, Gujarat, India

5 Minnesota Institute for Astrophysics, University of Minnesota, Minneapolis, MN 55455, USA

6 LESIA, Observatoire de Paris, CNRS UMR 8109, UPMC, Université Paris Diderot, 5 place J. Janssen, 92195 Meudon, France

7 Department of Physics and Astronomy, University College London, Gower Street, London, WC1E 6BT, UK

8 Astrophysics Research Institute, Liverpool John Moores University, Birkenhead, CH41 1LD, UK

9 Astrophysics Group, Keele University, Keele, Staffordshire ST5 5BG, UK

10 Space Science Applications Laboratory, The Aerospace Corporation, M2/266, PO Box 92957, Los Angeles, CA 90009, USA

11 Jodrell Bank Cent. for Astrophysics, School of Physics and Astronomy, Univ. of Manchester, Oxford Road, Manchester, M13 9PL, UK

Received 15 June 2012 / Accepted 17 July 2012

\begin{abstract}
Context. The fast temporal evolution of the ejecta morphology of novae can be considered as an important test bench for studying the shaping of many kinds of nebulae. V1280 Sco is one of the slowest dust-forming nova ever historically observed that has experienced a particularly long common-envelope phase.

Aims. We performed multi-epoch high-spatial resolution observations of the circumstellar dusty environment of V1280 Sco to investigate the level of asymmetry of the ejecta.

Methods. We observed V1280 Sco in 2009, 2010 and 2011 (from $t=877$ days after discovery until $t=1664 \mathrm{~d}$ ) using unprecedented high angular resolution techniques. We used the NACO/VLT adaptive optics system in the $J, H$ and $K$ bands, together with contemporaneous VISIR/VLT mid-IR imaging that resolved the dust envelope of V1280 Sco, and SINFONI/VLT observations secured in 2011. Results. We report the discovery of a dusty hourglass-shaped bipolar nebula. The apparent size of the nebula increased from $0.30^{\prime \prime} \times$ $0.17^{\prime \prime}$ in July 2009 to $0.64^{\prime \prime} \times 0.42^{\prime \prime}$ in July 2011 . The aspect ratio suggests that the source is seen at high inclination. The central source shines efficiently in the $K$ band and represents more than $56 \pm 5 \%$ of the total flux in 2009 , and $87 \pm 6 \%$ in 2011 . A mean expansion rate of $0.39 \pm 0.03$ milliarcsec per day is inferred from the VISIR observations in the direction of the major axis, which represents a projected upper limit. Assuming that the dust shell expands in that direction as fast as the low-excitation slow ejecta detected in spectroscopy, this yields a lower limit distance to V1280 Sco of $\sim 1 \mathrm{kpc}$; however, the systematic errors remain large due to the complex shape and velocity field of the dusty ejecta. The dust seems to reside essentially in the polar caps and no infrared flux is detected in the equatorial regions in the latest dataset. This may imply that the mass-loss was dominantly polar.

Conclusions. V1280 Sco is an excellent test case for studying the temporal evolution of dusty bipolar ejecta. As the nebula expands, observations will be easier and we advocate a yearly monitoring of the source using high angular resolution techniques.
\end{abstract}

Key words. techniques: high angular resolution - novae, cataclysmic variables - stars: individual: V1280 Scorpi - stars: mass-loss circumstellar matter

\section{Introduction}

White dwarfs (WDs) are the end products of the evolution of low- and intermediate-mass stars, with initial masses between 0.8 and $8 M_{\odot}$. If a white dwarf is in a close binary system, it can accrete hydrogen from its close companion's atmosphere, which then triggers the ignition of runaway nuclear reaction. This leads to a bright outburst, called a classical nova.

* Based on observations made with ESO telescopes at the Silla Paranal Observatory under programme IDs 283.D-5020, 385.D-0076, 087.D-0074.
The classical nova V1280 Sco was discovered in outburst by Yamaoka et al. (2007) on 2007 February 04.86 (JD = 2454136.85 ), about 12 days before reaching its maximum in visual light $\left(m_{\mathrm{V}} \sim 4\right)$ and formed dust only two weeks after reaching maximum in visible (Das et al. 2008). This nova experienced one of the slowest evolutions historically reported and the nebular phase was entered about 50 months $(t \sim 1600 \mathrm{~d})$ after outburst (Naito et al. 2012). This is a clear indication that the mass of the erupting WD is low (i.e. $\sim 0.6 M_{\odot}$ or even smaller).

Extensive monitoring of the event was performed using the Very Large Telescope Interferometer (VLTI) during the first four 
months (until $t=145 \mathrm{~d}$ ) of the event (Chesneau et al. 2008). Owing to the sparse $u v$ coverage, an interpretation involving a spherical dusty shell was developed, providing estimates of the dust formation rate. Based on an estimate of the total dust mass formed during the 250 days after outburst, Chesneau et al. (2008) suggested that a mass as large as $10^{-4} M_{\odot}$ was ejected. Naito et al. (2012) provided an independent estimate from neutral oxygen lines that confirmed the high mass ejected, an additional argument for a low-mass WD progenitor, known to statistically eject more mass that fast, massive ones. The interferometric observations also provided a consistent picture of the expansion rate in the plane of the sky estimated at $0.35 \mathrm{mas} / \mathrm{d}$. Radial velocities were published by Das et al. (2008) in the near-IR domain, and by Sadakane et al. (2010) in the visible. Sadakane et al. (2010) reported multiple high-velocity narrow components in the NaI D line from 650 to $900 \mathrm{~km} \mathrm{~s}^{-1}$. They interpreted this as being due to the formation of clumps, as a consequence of a strong shock between the slow, dust-forming ejecta, and a fast wind $\left(\sim 2000 \mathrm{~km} \mathrm{~s}^{-1}\right)$ generated during the second brightening of the source (Chesneau et al. 2008, $t=110 \mathrm{~d}$ ). P-Cygni lines were systematically observed even four years after the outburst, which is indicative of a sustained and significant mass loss (Schwarz et al. 2011). The typical P-Cygni type velocities reached $500 \mathrm{~km} \mathrm{~s}^{-1}$, from which Chesneau et al. (2008) inferred a distance of $1.6 \pm 0.4 \mathrm{kpc}$. However, Hounsell et al. (2010) recently derived a distance of $630 \pm 100$ pc from space-based visual light curves that span the first 20 days after discovery. This much smaller distance estimate demands that the VLTI-derived distance be scrutinized. Using the spatial expansion rate from the VLTI but a revised velocity range of the dust-forming ejecta of $350 \pm 160 \mathrm{~km} \mathrm{~s}^{-1}$ based on the mean velocity measured from blue-shifted absorption lines of OI and SiII, Naito et al. (2012) derived a revised distance of $1.1 \pm 0.5 \mathrm{kpc}$.

The dust event and the large amount of circumstellar material prevented any X-ray detection of V1280 Sco until May 2009, $t=834 \mathrm{~d}$ (Ness et al. 2009). After this date the source was detected several times from $t=834 \mathrm{~d}$ to $t=939 \mathrm{~d}$ after outburst (Schwarz et al. 2011), although the dense ejecta considerably absorbed the potentially important X-ray flux.

We report on observations of the Very Large Telescope (VLT) performed between $2009(t=857 \mathrm{~d})$ and $2011(t=$ $1664 \mathrm{~d})$ that reveal a striking bipolar nebula in expansion. The very slow ejecta of V1280 Sco likely expanded while being significantly influenced by the companion, although an intrinsically polar-oriented ejection scenario must also be investigated. This is a unique opportunity to observe a newly formed bipolar nebula. Our study has a broader relevance in the context of the shaping of asymmetrical bipolar nebulae and the formation of disks, such as that seen in some planetary or pre-planetary nebulae (Chesneau 2011; De Marco \& Soker 2011; Lykou et al. 2011; Chesneau et al. 2007), born-again sources (Lau et al. 2011; Chesneau et al. 2009) or in massive stars (Millour et al. 2011; Meilland et al. 2010; Kastner et al. 2010; Kraus et al. 2010) and for a better understanding of the still poorly known commonenvelope phenomenon (De Marco et al. 2011).

The observations are presented in Sect. 2. In Sect. 3 we derive some physical parameters of the nebula, and we discuss our results in Sect. 4.

\section{Observations}

The source was observed with the NACO, VISIR and SINFONI instruments of the VLT. The log of the observation is shown in Table 1 and the visible light curve is reproduced in Fig. 1.

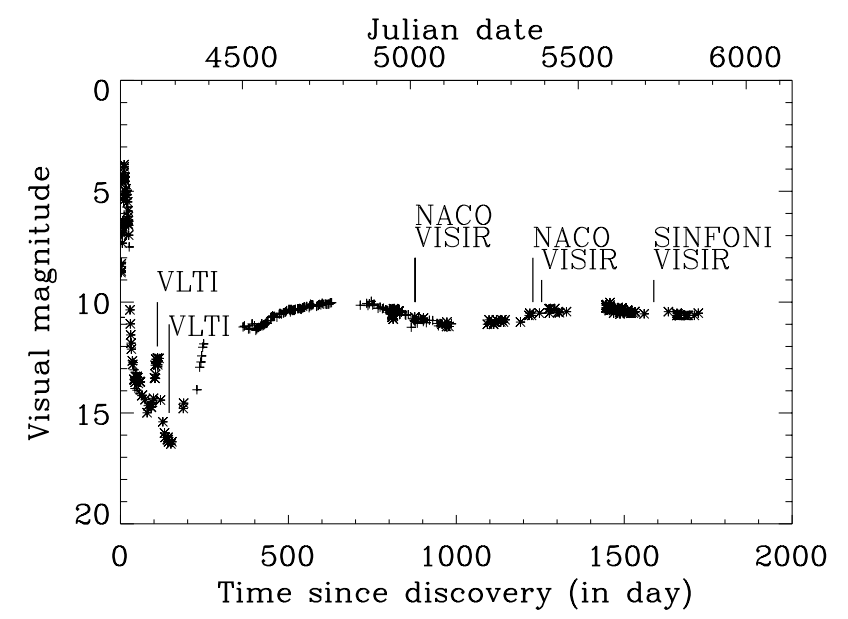

Fig. 1. Visual light curve of V1280 Sco from AFOEV and ASAS-3 data with the dates of the VLTI and VLT observations.

Table 1. NACO/VLT, VISIR/VLT and SINFONI/VLT observing logs.

\begin{tabular}{|c|c|c|c|c|}
\hline Date & Day $^{1}$ & $\mathrm{SCI} / \mathrm{CAL}$ & Inst. & Filters \\
\hline 2009.07 .02 & 877 & V1280 Sco & NACO & $J, H, K$ \\
\hline 2009.07 .02 & 877 & PSF $1^{2}$ & NACO & $J, H, K$ \\
\hline 2009.07 .03 & 878 & V1280 Sco & VISIR $^{4}$ & $N$ band filters 5 \\
\hline 2009.07.03 & 878 & $\mathrm{PSF} 2^{3}$ & VISIR $^{4}$ & $N$ band filters ${ }^{5}$ \\
\hline 2010.06 .17 & 1228 & V1280 Sco & $\mathrm{NACO}^{4}$ & $J, H, K$ \\
\hline 6.17 & 1228 & PSF $1^{3}$ & $\mathrm{NACO}^{4}$ & $J, H, K$ \\
\hline 2010.07 .28 & 1254 & V1280 Sco & VISIR $^{4}$ & $N$ band filters 5 \\
\hline 2010.07 .28 & 1254 & PSF $2^{3}$ & VISIR $^{4}$ & $N$ band filters 5 \\
\hline 2011.05 .21 & 1566 & V1280 Scc & SINFONI & $H, K$ \\
\hline 2011.06 .13 & 1589 & V1280 Sco & VISIR $^{4}$ & $N$ band filters 5 \\
\hline 2011.06 .13 & 1589 & PSF $2^{3}$ & VISIR $^{4}$ & $N$ band filters 5 \\
\hline 2011.06.26 & 1602 & V1280 Sco & SINFONI & $K$ \\
\hline 2011.06 .27 & 1603 & V1280 Sco & SINFONI & $K$ \\
\hline 2011.07.03 & 1609 & V1280 Sco & SINFONI & $H, K$ \\
\hline 2011.07 .03 & 1609 & V1280 Sco & SINFONI & $H, K$ \\
\hline 2011.08.26 & 1663 & V1280 Sco & SINFONI & $H, K$ \\
\hline 2011.08.28 & 1664 & V1280 Sco & SINFONI & $H, K$ \\
\hline
\end{tabular}

Notes. ${ }^{(1)}$ Day after outburst. ${ }^{(2)}$ GSC 07364-01316, $J=8.29 \pm 0.03$, $H=7.68 \pm 0.07,7.48 \pm 0.02$. ${ }^{(3)} \mathrm{HD} 152934, \mathrm{M} 5 \mathrm{~V}, V=11, K=2.46$, IRAS f1 $2=8.19$ Jy. ${ }^{(4)}$ Burst mode. ${ }^{(5)}$ PAH1, $\lambda=8.59 \mu \mathrm{m}, \Delta \lambda=$ $0.42 \mu \mathrm{m}$; ArIII, $\lambda=8.99 \mu \mathrm{m}, \Delta \lambda=0.14 \mu \mathrm{m}$; PAH2, $\lambda=11.25 \mu \mathrm{m}$, $\Delta \lambda=0.59 \mu \mathrm{m}$; PAH2_2, $\lambda=11.88 \mu \mathrm{m}, \Delta \lambda=0.37 \mu \mathrm{m}$; NeII, $\lambda=$ $12.81 \mu \mathrm{m}, \Delta \lambda=0.21 \mu \mathrm{m} ;$ NeII_1, $\lambda=12.27 \mu \mathrm{m}, \Delta \lambda=0.18 \mu \mathrm{m}$.

\subsection{NACO observations}

We observed V1280 Sco in $J, H, K$ s with the S13 camera, which provides a field of view (FOV) of $14^{\prime \prime} \times 14^{\prime \prime}$ and a pixel scale of $13.26 \pm 0.03$ mas. The AutoJitter mode was used in which the telescope moves according to a random pattern in a $5^{\prime \prime}$ box at each exposure. The nearby star GSC 07364-01316 (Lasker et al. 2008) was selected as the reference star for the visible wavefront sensor of the adaptive optics (AO) for determining the point spread function (PSF) of the instrument and to serve as a rough flux calibrator. The PSF obtained in 2009 with NACO in standard mode was not good enough to allow a meaningful deconvolution of the compact source (Fig. 2). In 2010, the NACO observations benefited from the newly implemented "cube mode", which provides a large set of short exposures (30 ms) over part of the detector. 8000 frames were recorded per 
O. Chesneau et al.: A bipolar dusty nebula around the nova V1280 Sco
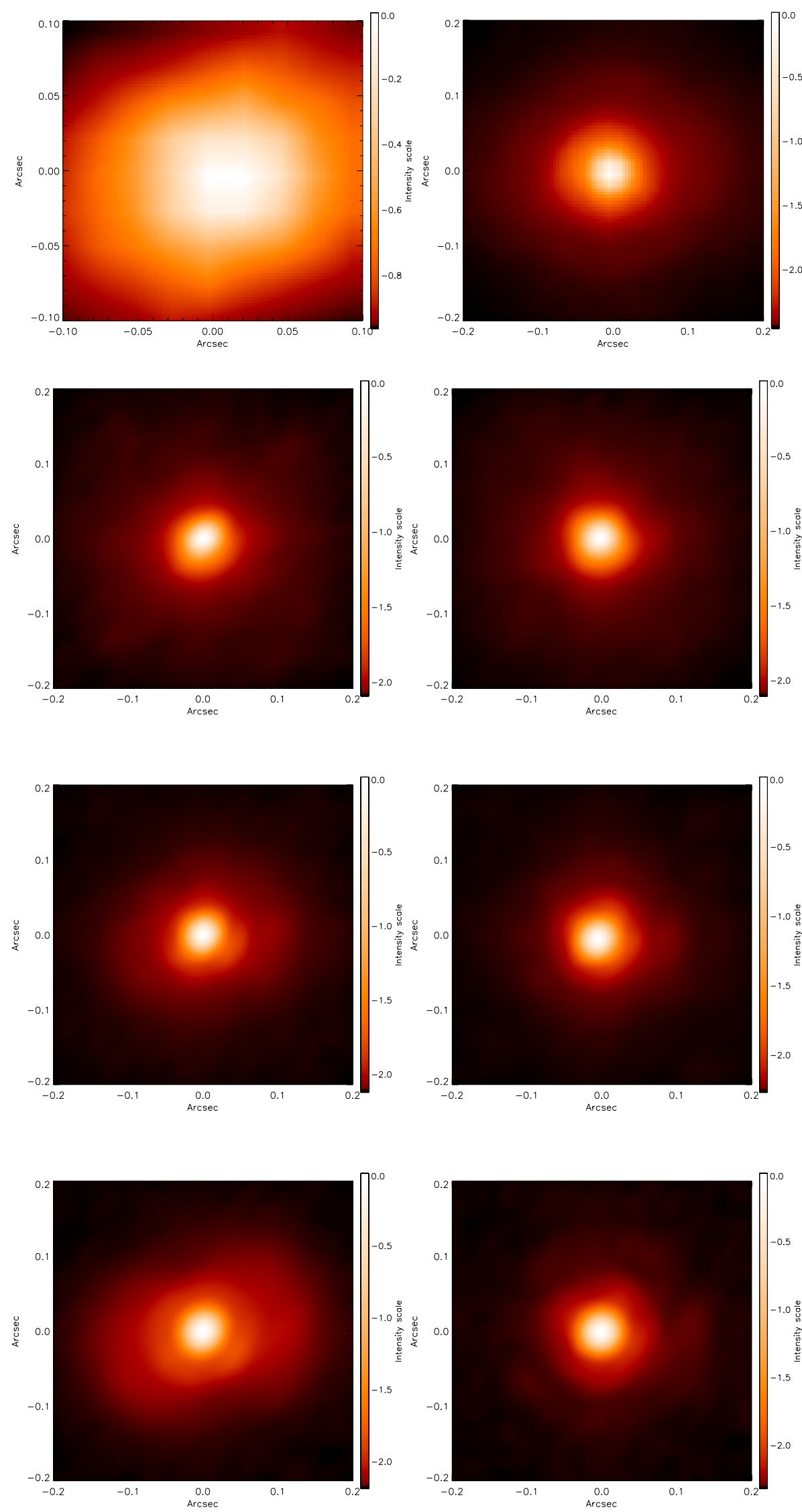

Fig. 2. Left: normalized $2009 K$ band NACO image of V1280 Sco in logarithmic scale. Right: same for the calibrator.
Fig. 3. Upper left: normalized $2010 \mathrm{~J}$ band NACO image of V1280 Sco in logarithmic scale. Upper right: $2010 J$ band NACO image of the calibrator. Middle: same in the $H$ band. Bottom: same in the $K$ band. A significant brightening of the central source (unresolved) is observed compared to 2009 (Fig. 2). filter, representing an exposure time of $480 \mathrm{~s}$ each (Figs. 3 and 4). This new capability is essential to reach truly diffraction-limited imaging at the shortest wavelengths (Kervella et al. 2009). The cube mode provides a large cube of short exposures from which a sub-set with the highest Strehl ratio is selected (principle of "lucky imaging"). 
A\&A 545, A63 (2012)

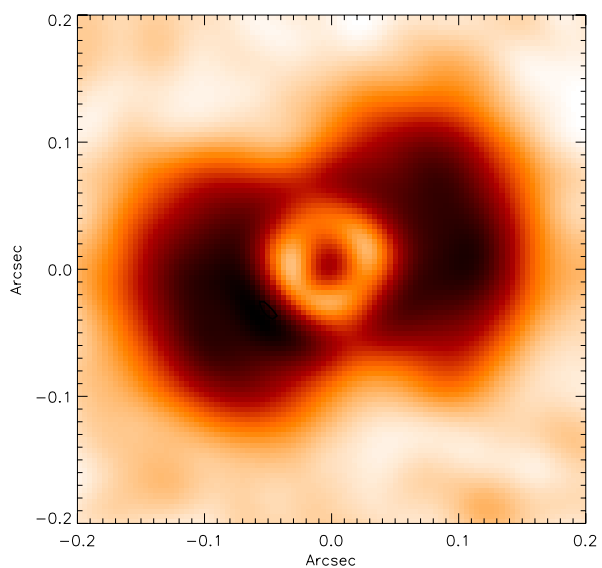

Fig. 4. 2010 NACO $K$ band image after a PSF subtraction. The color table is inverted.

\subsection{VISIR observations}

The NACO observations were complemented by mid-infrared images obtained with the VISIR instrument (Lagage et al. 2004). The VISIR observations were performed in July 2009 (MJD 55014.2 and 55 015.1, $t=877-878 \mathrm{~d}$ ), June and July 2010 $(t=1228 \mathrm{~d}$ and $t=1254 \mathrm{~d})$ and June $2011(t=1589 \mathrm{~d})$. The atmospheric conditions were stable for all nights. The imager was used in burst mode (DIT ranging from 10 to $25 \mathrm{~ms}$ ), providing a pixel scale of $0.075^{\prime \prime}$ and a field of view of $19.2^{\prime \prime} \times$ $19.2^{\prime \prime}$. The chopping throw was 8 arcsec and the chopping frequency $0.25 \mathrm{~Hz}$. A set of narrow-band filters covering the full $N$ band were used, with integrated total exposure times per filter ranging from $280 \mathrm{~s}$ to $360 \mathrm{~s}$. Several filters were centered on potential spectral features: PAH1, PAH2 and NeII. The exposures taken with the PAH2 $(\lambda=11.25 \mu \mathrm{m}, \Delta \lambda=0.59 \mu \mathrm{m})$ and NeII filters $(\lambda=12.81 \mu \mathrm{m}, \Delta \lambda=0.21 \mu \mathrm{m})$ were immediately followed by exposures taken with a nearby continuum filter called PAH2_2 $(\lambda=11.88 \mu \mathrm{m}, \Delta \lambda=0.37 \mu \mathrm{m})$ and NeII_1 $(\lambda=12.27 \mu \mathrm{m}, \Delta \bar{\lambda}=0.18 \mu \mathrm{m})$.

The standard chopping/nodding technique was applied to remove the sky contribution. The burst mode provides a large cube of short-exposure frames in which all single chopping and nodding images recorded. The reduction of the VISIR data additionally includes the correction of various defects exhibited by the VISIR detector.

The VISIR burst mode and NACO cube modes are very similar. The principle is to acquire very short exposures $(\leq 50 \mathrm{~ms})$ to keep the complete integration within a fraction of the coherence time $(\sim 300 \mathrm{~ms}$ at Paranal in the mid-IR). The burst modes of NACO and VISIR provided large cubes of short exposures that enable the reconstruction of quality-enhanced images using shift-and-add techniques, and selecting the best percentile (lucky imaging) following an algorithm described by Kervella et al. (2009) and Lagadec et al. (2011). The VISIR burst mode images of V1280 Sco were deconvolved using a Richardson-Lucy algorithm. Thirty iterations were sufficient to obtain good quality converged images. The VISIR images are shown in Fig. 5 (2009), Fig. 6 (2010) and Fig. 7 (2011).

Flux calibration was performed using standard aperture photometry methods applied to the science and reference stars. The observations were performed in fairly similar atmospheric conditions and the raw fluxes observed per filter using the same calibrator are similar at better than 5\%. Unfortunately, the VISIR PSF calibrator, HD 152934, was chosen for its brightness but was a poorly suited photometric calibrator. HD 152934 is an
Table 2. Extensions of the bipolar nebulae.

\begin{tabular}{lccc}
\hline \hline Filter & Major axis $^{1}$ & Minor axis & PSF $F W H M$ \\
\hline & & 2009 & \\
$K$ & $0.29 \pm 0.04^{\prime \prime}$ & $0.17 \pm 0.03^{\prime \prime}$ & $0.092 \pm 0.005^{\prime \prime}$ \\
PAH2 & $0.31 \pm 0.04^{\prime \prime}$ & - & $0.23 \pm 0.03^{\prime \prime}$ \\
\hline \multicolumn{3}{c}{2010} \\
$K$ & $0.40 \pm 0.03^{\prime \prime}$ & $0.26 \pm 0.02^{\prime \prime}$ & $0.057 \pm 0.003^{\prime \prime}$ \\
PAH2 & $0.49 \pm 0.05^{\prime \prime}$ & $0.33 \pm 0.05^{\prime \prime}$ & $0.23 \pm 0.03^{\prime \prime}$ \\
\hline & & 2011 \\
$K$ & $0.62 \pm 0.05^{\prime \prime}$ & $0.39 \pm 0.01^{\prime \prime}$ & $0.13 \pm 0.02^{\prime \prime}$ \\
PAH2 & $0.64 \pm 0.06^{\prime \prime}$ & $0.37 \pm 0.05^{\prime \prime}$ & $0.23 \pm 0.03^{\prime \prime}$ \\
\hline
\end{tabular}

Notes. ${ }^{(1)}$ Corrected for the FWHM of the PSF.

anonymous S-type AGB star whose spectral type is not well constrained. We used synthetic templates of S-type stars produced by the MARCS codes (Gustafsson et al. 2008) to fit the SED of HD 152934. We then estimated the flux in the VISIR filters using the best synthetic spectrum $\left(T_{\text {eff }}=3000 \mathrm{~K}\right.$ and $\left.\log g=0\right)$ and calibrated the science object photometrically. This procedure is indirect and leads to a potential calibration bias larger than $10 \%$ in some filters for such a complex source.

\subsection{SINFONI observations}

VLT/SINFONI is a near-infrared (1.1-2.45 $\mu \mathrm{m})$ integral field spectrograph (IFS) fed by an AO module (Eisenhauer et al. 2003). Integral field spectroscopy allows one to gather spectra of the sky over a two-dimensional field of view. Final products are data cubes with two dimensions of $x, y$ and a third dimension formed by the wavelengths. During our observations the spectrograph operated in the $K$ and $H$ bands, providing a spectral resolution of 4000 and 3000 , respectively. The FOV was cut into 32 slices, and each slice was projected onto 64 detector pixels. The field of view of the data set is $0.8^{\prime \prime} \times 0.8^{\prime \prime}$ and is composed of spaxels of $12.5 \times 25$ mas in the $K$ and $H$ bands. We obtained several VLT/SINFONI integral field spectroscopic data sets for V1280 Sco from May to August 2011. The FOV was oriented such that the major axis of the nebula roughly coincided with the vertical direction. During that period, the central stellar-like source increased considerably in flux, while the bipolar nebula in which this source is embedded had a spatial extent of $\leq 0.7^{\prime \prime}$ which made these observations challenging. The observations were performed in service mode and were repeated several times, but the atmospheric conditions never met the criteria for providing good quality data. We nevertheless worked on the best data of the sample and present the most robust results. The data set of 26 June 2011 exhibited the better signal-to-noise ratio $(\mathrm{S} / \mathrm{N})$ although the bipolar nebula was slightly cut in the north. Because of the limited $\mathrm{S} / \mathrm{N}$ of the observations and the difficult removal of the telluric lines, a differential approach, using a comparison between a given emission line flux and its corresponding adjacent continuum was employed in the reduction and analysis.

\section{Observational results}

\subsection{The dusty nebula}

A bipolar nebula with a high aspect ratio is clearly seen in the NACO, SINFONI and VISIR images we obtained, suggesting that the source is seen at high inclination. For our NACO and SINFONI data, the nebula extension was estimated by determining the regions of images with the highest gradient before 

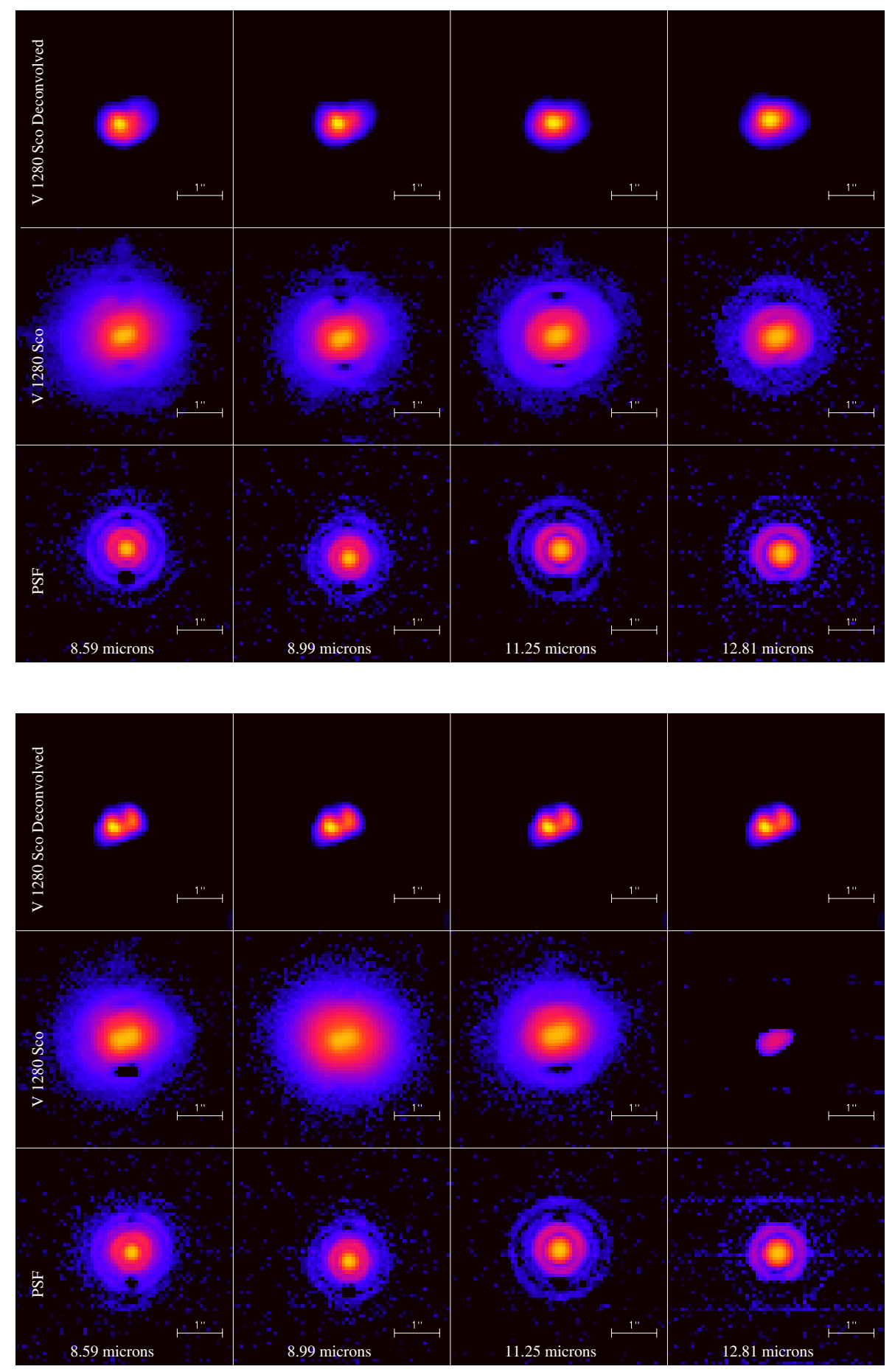

Fig. 5. Burst-mode VISIR observations performed in $2009(t=878 \mathrm{~d})$ with four different filters (see description in Table 1). Up: deconvolved images of V1280 Sco. Middle: mean images generated from the best short exposures of V1280 Sco. Bottom: mean images of the PSF with the same treatment.
Fig. 6. Same for the VISIR observations performed in $2010(t=1254 \mathrm{~d})$. reaching the noise level (or written differently, by the regions of densest contour levels before reaching the noise level). The estimation was performed on the images with and without PSF subtraction. The extensions measured are reported in Table 2.

The near-IR images were dominated by the central source, which brightened considerably over the three years of observations. The apparent size in the $K$ band (Fig. 2) was $304 \times 191$ mas in $2009(t=877 \mathrm{~d})$. Corrected for the PSF extension, this leads to an angular size of $290 \pm 38 \times 167 \pm 31$ mas. After a PSF subtraction, the position angle (PA) of the major axis is as $105 \pm 5^{\circ}$ with an axial ratio as $\sim 1.7$. However a good estimate of the minor axis extension was difficult to obtain due to the bright central source, and the artifacts remaining after the PSF subtraction.
The NACO observations performed in cube mode in 2010 ( $t=1228 \mathrm{~d}$ ) were of excellent quality, and the $J, H$ and $K$ bands can be fully exploited (Figs. 3 and 4 ). An elongation was clearly detected in each image at $\mathrm{PA}=105 \pm 3^{\circ}, 102 \pm 3^{\circ}$ and $107 \pm 3^{\circ}$. For instance, the FWHM of the $J$ band image of V1280 Sco is $61 \pm$ 3 mas along the major axis, and $54 \pm 3$ along the minor axis. The apparent size in the $K$ band was $404 \times 260$ mas, which, corrected for the FWHM of the PSF, corresponds to $400 \pm 30 \times 255 \pm 24$ mas.

The 2011 SINFONI images $(t=1602 \mathrm{~d})$ suggest that the source is composed of a bright unresolved source and two apparently well-detached polar caps. There seems to be no indication for extended equatorial material. The extension of the nebula in the direction of the major axis is estimated to be $637 \pm 13$ mas, 


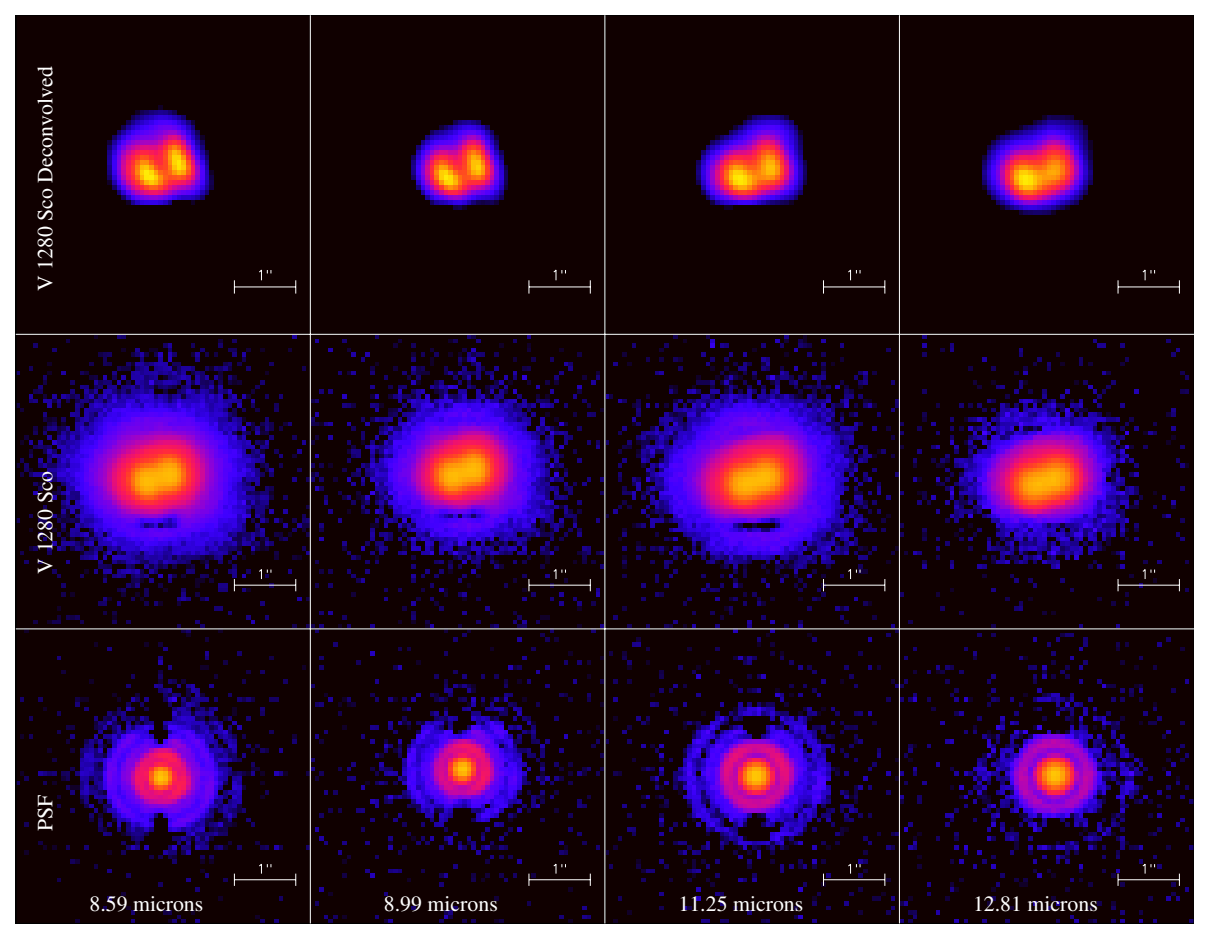

Fig. 7. Same for the VISIR observations performed in $2011(t=1589 \mathrm{~d})$.
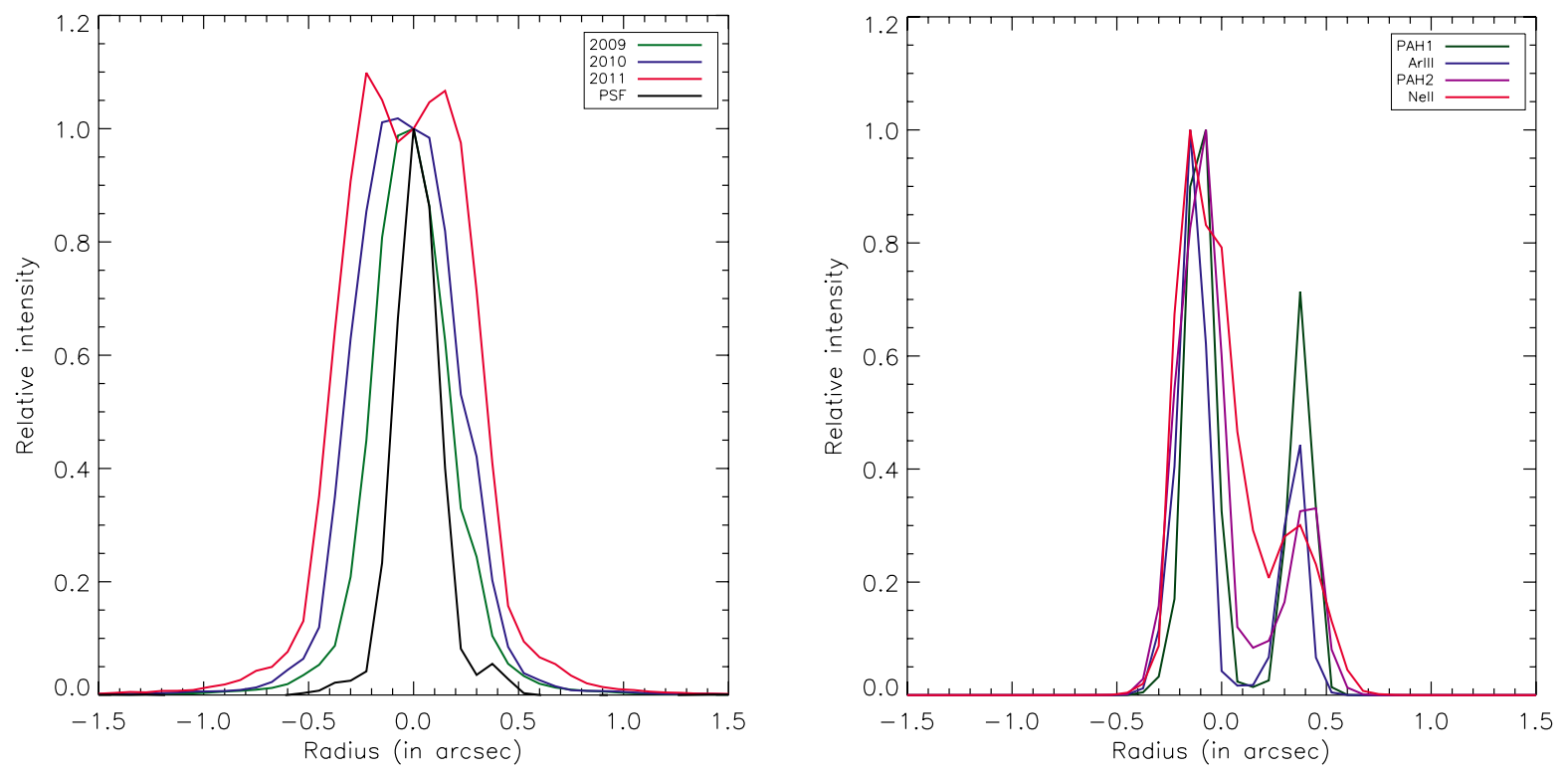

Fig. 8. Left: cut of the VISIR raw images at $8.59 \mu \mathrm{m}$ along the major axis of the dust shell for the three epochs. An expansion of the dusty material is clearly seen. Right: cut of the VISIR 2011 deconvolved images along the main axis of the dust shell for all filters.

and the larger extent of the caps perpendicular to the major axis to be $412 \pm 13$ mas (Fig. 9 and Table 2).

The VISIR images also provided robust estimates of the PA at $108.5 \pm 2^{\circ}$ in $2009,109.1 \pm 1.6^{\circ}$ in 2010 and $108.9 \pm 2.5^{\circ}$ in 2011. The bipolar morphology of our VISIR images was more clearly seen in the most recent images due in part to the expansion of the nebula. Figure 8 (left) shows a cut of the VISIR images obtained at $8.59 \mu \mathrm{m}$ in 2009, 2010 and 2011, together with a PSF for comparison. This filter provides the shortest wavelength, hence the best spatial resolution of VISIR and was therefore used for providing the extension estimates (Table 2). The bipolar lobes of V1280 Sco are clearly expanding with time, and in 2011, one could see a double peaked cut generated by the two emission peaks of the bipolar nebula. One-dimensional Gaussian fitting was performed to measure the FWHM of the cuts, and the results are shown in Table 2. After deconvolution, the VISIR images provide a size and an axial ratio equivalent to that seen in the near-IR. The size and appearance of the nebula extension was nearly unchanged from 8 to 13 micron. The deconvolved images confirm that the object is bipolar and expanding. Our most resolved images (from our 2011 dataset) show that the intensities of the two bipolar lobes are different, the southern lobe is brighter. This is probably due to an absorption effect as a consequence of this lobe being closer to us than the northern one. No mid-IR emission is seen between the caps. Figure 8 shows a radial cut along the poles at 8.59.8.99, 11.25, and $12.81 \mu \mathrm{m}$. The separation between the two lobes does not depend on the wavelength. The ratio of the intensity between the two peaks increases with wavelength due to absorption and optical depth effects. 

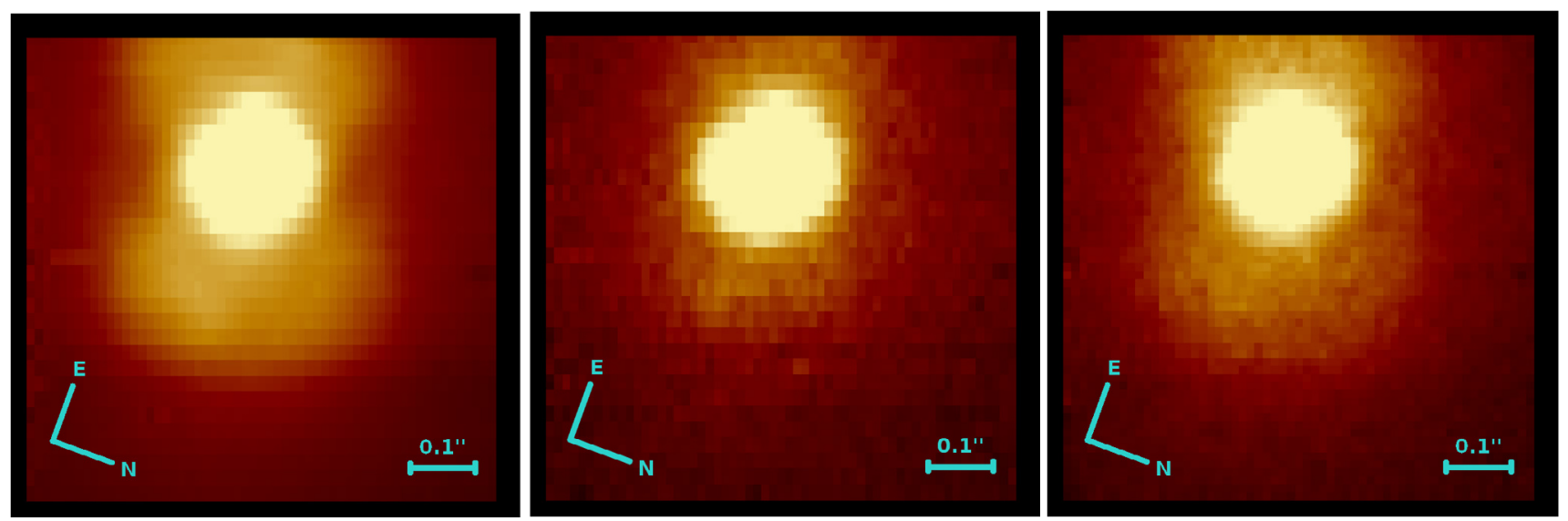

Fig. 9. Left: continuum flux from a narrow spectral channel $(2.7 \mathrm{~nm})$ close to the $\mathrm{Br} \gamma$ line. Middle: same from a channel centered on the Br $\gamma$ line emission from which the nearby continuum shown on the left was removed. Right: same from a channel centered on the HeI2.05 line emission isolated from a nearby continuum. The $\mathrm{Br} \gamma$ line forming region is centered on the outbursting source, whereas some intrinsic HeI2.05 emission seems to originate from the lobes.

Table 3. Photometry of the total source.

\begin{tabular}{lcccc}
\hline \hline Filter & $\begin{array}{c}\lambda_{0} \\
{[\text { micron] }}\end{array}$ & $\begin{array}{c}t=878 \mathrm{~d} \\
{[\mathrm{Jy}]}\end{array}$ & $\begin{array}{c}t=1254 \mathrm{~d} \\
{[\mathrm{Jy}]}\end{array}$ & $\begin{array}{c}t=1589 \\
{[\mathrm{Jy}]}\end{array}$ \\
\hline$J$ & 1.05 & - & $0.38 \pm 0.07$ & - \\
$H$ & 1.65 & - & $0.44 \pm 0.04$ & - \\
$K$ & 2.1 & $0.67 \pm 0.04$ & $0.44 \pm 0.02$ & - \\
PAH 1 & 8.59 & $47 \pm 6$ & $34 \pm 4$ & $28 \pm 4$ \\
ArIII & 8.99 & $43 \pm 5$ & $32 \pm 4$ & $26 \pm 3$ \\
PAH 2 & 11.25 & $33 \pm 3$ & $25 \pm 3$ & $20 \pm 3$ \\
PAH 2_2 & 11.88 & $31 \pm 4$ & $23 \pm 3$ & $19 \pm 3$ \\
NeII & 12.81 & $26 \pm 3$ & $20 \pm 3$ & $16 \pm 2$ \\
\hline
\end{tabular}

Notes. ${ }^{(1)}$ Filter dedicated to continuum measurements close to the $\mathrm{PAH} 2$ filter.

\subsection{Spectroscopy and flux measurements}

The $V$ flux has reached a roughly stable plateau at a magnitude of about 10.5 over a long period (Naito et al. 2012) and the central source radiates efficiently in the $K$ band. As shown by Lynch et al. (2009), the nova is still powering a strong wind, and extensive nuclear burning is thus thought to have continued.

An unresolved point source dominates the near-IR observations, whereas no point source contribution is observable in the mid-IR for the contemporaneous VISIR measurements. In 2009, the stellar source (i.e. the unresolved flux) accounts for $54 \pm 6 \%$, increasing in 2010 to reach $76 \pm 6 \%$ (NACO data) and in 2011 $87 \pm 5 \%$ (SINFONI) of the total flux. These numbers are potentially biased by the different Strehl ratio reached by the AOs during the observations. These estimates were also possible in the $H$ and $J$ bands for the 2010 NACO observations, with $96 \pm 3 \%$ and $100 \pm 3 \%$, respectively. This increase of the central source contribution indicates a decrease of the circumstellar absorption, related probably to the expansion and dissipation of the nearby dust.

The photometry of the central source and its nearby environment was performed from the NACO, SINFONI and VISIR images and is provided in Table 3 . These instruments or the mode used are not necessarily optimized to provide accurate photometry but despite the level of errors such information remains of high interest for these variable sources.
Many lines are detected by SINFONI $(t=1609 \mathrm{~d})$. Of importance is the simultaneous detection of relatively high-excitation lines (mostly from the Balmer series), and the detection of low ionization species such as $\mathrm{NaI}$ and $\mathrm{MgI}$, as already reported by Das et al. (2008) and Sadakane et al. (2010) for this source. The mean FWHM of the Balmer lines is $162 \pm 14 \mathrm{~km} \mathrm{~s}^{-1}$, and we note that $\mathrm{Br} \gamma$ exhibits a broad pedestal $\left(\sim 700 \mathrm{~km} \mathrm{~s}^{-1}\right)$. Some lines of low excitations are observed, the NaI 2.149 line $(F W H M=$ $\left.110 \pm 18 \mathrm{~km} \mathrm{~s}^{-1}\right), \operatorname{MgI} 1.711\left(F W H M=144 \pm 8 \mathrm{~km} \mathrm{~s}^{-1}\right)$, some are unidentified such at $2.137 \mu \mathrm{m}\left(F W H M=105 \pm 8 \mathrm{~km} \mathrm{~s}^{-1}\right)$ and some with weak signatures such as MgI 1.576, CI 1.690, as reported by Das et al. (2008). These lines are narrow and almost unresolved given the spectral resolution of these observations $\left(R \sim 3500\right.$ corresponding to a $F W H M$ of $85 \mathrm{~km} \mathrm{~s}^{-1}$ ). The presence of the NaI 2.149 line implies that the NaI must be protected from the central source, and is therefore very likely located within the dust. The presence of these low-excitation lines so late $(t=1609 \mathrm{~d})$ after the outburst highlights how slowly V1280 Sco has evolved, because these lines are not expected at this stage.

For the strongest lines, a SINFONI image was created by integrating the flux within a narrow spectral range, and by subtracting a red- and blue-sided continuum. For the Br $\gamma$ line, the main source of the emission is observed in the central object, which contributes $98 \pm 7 \%$ of the total flux. A slightly different behavior may be observed for the He I $2.06 \mu$ m (see Fig. 9) line for which the core contributes $93 \pm 7 \%$, and for which the lobes seem better contrasted. In the case of V1280 Sco, one may hypothesize that the He I $2.06 \mu \mathrm{m}$ emission in the caps could arise either from a shock between the fast nova wind and the slowly expanding, dust rich polar caps, or by a radiative interaction between this material and the growing UV flux from the white dwarf.

The PAH_2 filter is designed to detect carbonaceous molecules. This filter is not best suited because the $11.25 \mu \mathrm{m}$ feature in novae is seen at $\sim 11.4 \mu \mathrm{m}$ (Evans et al. 2005), although most of the flux should be isolated by the filter given the bandwidth $(0.59 \mu \mathrm{m})$. Yet, it is challenging to detect any morphological difference between images in the two filters because of the limited spatial resolution and the weakness of the flux contrast. It is not evident that the regions of PAH emission is distinct from that of the continuum. Spitzer IRS (6 May 2008) and IRTF/Spex (20 May 2009) observations show that dust emission 
features appeared at 3.28, 3.4, 8.3, 8.7, and 11.4 microns, the socalled UIRs (unidentified infrared bands). Likely, carbonaceous molecules also formed in situ from condensed dust (Bernstein \& Lynch 2009; Evans et al. 2010, 2005, HAC).

\subsection{The expansion rate}

In the near-IR, the datasets are not homogeneous with images taken with NACO in 2009 without cube mode, then in 2010 in cube mode and in 2011 using the SINFONI instrument. We did not attempt to derive an expansion rate for the lobes in the $K$ band due to the variances in the observational techniques. Despite their lower spatial resolution, the images from the VISIR instrument were obtained solely in burst mode. Moreover, in the mid-IR, the central source is too faint to be detected, and the images probe the contribution from the dust alone. The NACO and SINFONI images exhibit a bright stellar contribution that has to be carefully taken into account and removed before any geometrical information can be inferred. The expansion was measured from the raw and deconvolved VISIR images. Figure 8 shows the cuts of the images along the direction of the major axis and the results are shown in Table 2. Correcting the FWHMs from the FWHM of the PSF at $8.59 \mu \mathrm{m}$, this leads to an estimate of the expansion rate of $0.36 \pm 0.06$ (2009), $0.39 \pm 0.05$ (2010) and $0.40 \pm 0.04$ (2011) mas per day, from which we infer a global estimate of $0.39 \pm 0.03$ mas per day. As an independent test, one can compute the separation of the polar caps as measured by SINFONI to be $0.62 \pm 0.05^{\prime \prime}$ at day 1602 , providing an expansion rate very close to the VISIR measurements.

This value, measured in the direction of the major axis of the nebula is close to the expansion rate estimate of $0.35 \pm$ 0.03 mas per day from the VLTI in a similar wavelength range. The VLTI baselines used to infer the expansion of the early dusty envelope were in a direction close to the major axis of the nebula (within typically $30^{\circ}$ ), and therefore approximately probed the velocities projected on the sky in that direction. The bias expected from the use of baselines not aligned to the major axis is an underestimate of the expansion rate.

\section{Discussion}

\subsection{The distance and expansion velocities}

Using the angular expansions measured from the infrared images, one can try to improve upon the distance estimate for V1280 Sco. The angular expansion inferred from the VLTI observations was $0.35 \pm 0.03 \mathrm{mas} / \mathrm{d}$, while the high-quality VISIR images obtained in the study suggest a slightly higher expansion rate in the direction of the major axis of the nebula of $0.39 \pm 0.03 \mathrm{mas} / \mathrm{d}$.

Combined with a measure of the ejecta expansion velocity, the angular expansion rate (mas/d) can be used to derive a distance to V1280 Sco, under the hypothesis of a spherical ejection following

$D_{\mathrm{kpc}}=1.154 \times 10^{-3} \frac{V_{\mathrm{ej}}}{\frac{\mathrm{d} \theta}{\mathrm{d} t}}$,

where $V_{\text {ej }}$ is the velocity of the ejecta in $\mathrm{km} \mathrm{s}^{-1}$ and $\frac{\mathrm{d} \theta}{\mathrm{d} t}$ is the rate of expansion in mas per day.

Assuming a velocity $V_{\mathrm{ej}}$ for the ejecta of $500 \pm 100 \mathrm{~km} \mathrm{~s}^{-1}$, Chesneau et al. (2008) estimated the distance to be $1.6 \pm 0.4 \mathrm{kpc}$.
The difficulty of this exercise is to ensure that the Doppler velocities inferred from the emission lines can be linked to the dust expansion measured in the VLTI data or VISIR images. These quantities must be measured at the same wavelengths, if possible centered on an emission line that provides the kinematics. Moreover, the Doppler velocities are translated into ejection velocities assuming a projection factor (the $\mathrm{p}$ factor) whose value depends on the kinematics but also on the flux distribution of the source.

The discovery of the strong bipolarity of the source implies a complex velocity field that cannot be directly related to a limited number of velocity measurements. The expansion velocities were measured in the direction of the major axis of the bipolar nebula and we adopted a Doppler velocity of $500 \mathrm{~km} \mathrm{~s}^{-1}$, based on several radial velocity measurements from various emission lines. Co-mixing of the dust and gas emitting regions cannot be unequivocally demonstrated, and it is unknown whether the line-forming region coincides with the dust-continuum-forming region. Therefore, it is very difficult to measure the ejection velocity of the dusty nebula.

Hounsell et al. (2010) derived a much closer distance estimate, $630 \pm 100 \mathrm{pc}$ based on various estimates of intrinsic luminosity of the source. However, despite the quality of their photometry, many parameters are used to infer the distance, some of them raised to high power indices, implying potentially large errors. Hounsell et al. (2010) found $M_{\mathrm{v}}=-6.1$, yielding a distance of $\leq 1 \mathrm{kpc}$; however, they pointed out that $M_{\mathrm{v}}$ can be equal to -8 based on $t_{3}$ estimated as $34 \mathrm{~d}$, where $t_{3}$ is the number of days in which a nova wanes by 3 mag after maximum. This value yields a distance of $1.4 \mathrm{kpc}$, which is within the uncertainty of the first estimate from Chesneau et al. (2008).

If one assumes the distance quoted by Hounsell et al. (2010), the mean dust-forming region velocity is only $\sim 220 \mathrm{~km} \mathrm{~s}^{-1}$. This velocity is the velocity measured in the plane of the sky, in the direction of the major axis. Owing to the high aspect ratio, a high inclination is expected, and this measurement should be considered as close to the upper limit of the velocity field, and one may consider whether such a slowly expanding material may imprint its signature in spectroscopic observations.

Sadakane et al. (2010) reported on spectroscopic information of great interest to this problem. They observed absorption in the NaI lines detected between $-649 \mathrm{~km} \mathrm{~s}^{-1}$ to $-885 \mathrm{~km} \mathrm{~s}^{-1}$ with the strongest components at -750 and $-800 \mathrm{~km} \mathrm{~s}^{-1}$. One may assume that these absorption features provide a good approximation of the dust velocity field because they probe lowexcitation cool regions. Das et al. (2008) detected many lines from these low-ionization species ( $\mathrm{NaI}$ and $\mathrm{MgI})$ at the earliest stages of the event (i.e. at the onset of the dust-formation phase). The impressive narrowness of the features $\left(\sim 15 \mathrm{~km} \mathrm{~s}^{-1}\right)$ can be interpreted as originating from a cool medium. The measured FWHM can be considered as an upper limit, accounting for the instrumental FWHM of $\sim 5 \mathrm{~km} \mathrm{~s}^{-1}$. Hence, accounting for some turbulence and velocity dispersion of the expanding material in the FWHM broadening, the thermal broadening may originate from material cooler than $1500 \mathrm{~K}$. The Sadakane et al. (2010) observations and the 2009 NACO/VISIR images were obtained almost at the same epoch in mid 2009. The slower absorbing material in the line of sight was moving in 2009 at a velocity of about $650 \mathrm{~km} \mathrm{~s}^{-1}$. Naito et al. (2012) reported on much lower velocities, $350 \pm 160 \mathrm{~km} \mathrm{~s}^{-1}$, measured from blue-shifted absorption lines of OI and SiII. These low velocities were measured around light-curve maximum and increased up to $\sim 500 \mathrm{~km} \mathrm{~s}^{-1}$ just before the dust-formation phase. Using the expansion of VISIR of $0.39 \pm 0.03 \mathrm{mas} / \mathrm{d}$ in the major axis direction and the 
slowest velocities of the cool material of $350 \mathrm{~km} \mathrm{~s}^{-1}$, one still derives a distance estimate close to $1.0 \mathrm{kpc}$. This value is a lower estimate, since the velocities in the direction of the major axis of the bipolar nebula are expected to be higher, while the spectroscopically detected absorption components are seen in the line-of-sight direction. Lacking an accurate estimate of the inclination, we are left with this estimate which is potentially affected by larger errors.

To conclude, despite the significant limitations and errors that may affect the distance estimate of $1.6 \mathrm{kpc} \pm 0.4$ provided by Chesneau et al. (2008), we are reasonably confident that the distance of V1280 Sco is much larger than the distance of $0.6 \mathrm{kpc}$ proposed by Hounsell et al. (2010).

\subsection{Origin of the bipolarity}

A growing body of observational evidence clearly suggests that many nova ejectae are highly bipolar in the first days and months after the outburst. The correlation between speed class and ejection velocity, meaning that the faster the expansion speed, the less shaped the remnant (Bode 2002; Downes \& Duerbeck 2000), is an argument for supporting the significant effect of the common envelope phase on the shaping. The ejecta from a slow nova would feel the influence of the secondary much longer, leading to a strong common envelope interaction. These effects, involving frictional deposition of energy and angular momentum from the secondary are described by Kato \& Hachisu (2011); Porter et al. (1998); Lloyd et al. (1997); Livio et al. (1990). The very slow nova HR Del (Moraes \& Diaz 2009; Harman \& O'Brien 2003; Solf 1983, $t_{3}=230$ d) or V723 Cas (Lyke \& Campbell 2009; Evans et al. 2003, $t_{3}=180$ d) are striking examples of this phenomenon.

V1280 Sco appears to be at odds with the $t_{3}$ /axial ratio relationship shown for instance in Fig. 6 of Bode (2002). The axial ratio of V1280 Sco is high, surely higher than 1.5. Such high axial ratios are exhibited by the slowest novae in the diagram, with $t_{3}>100 \mathrm{~d}$. Hounsell et al. (2010) estimated $t_{3}=34$ days, yet cautioned that the fidelity of this determination is weak owing to the complexity of the light curve, which is affected by the dust and the secondary maximum. We cannot exclude that $t_{3}$ might have been shorter, although given the distance estimate of $1.6 \mathrm{kpc}$ that is favored in our study, $t_{3}=34 \mathrm{~d}$ fits the maximum magnitude-rate of decline relation (MMRD) relatively well (Downes \& Duerbeck 2000).

The late SINFONI images suggest that the dust resides only in the polar caps, and that the equatorial regions seem devoid of any dust in contrast to the bipolar helium nova V445 Pup for instance, which exhibits a large and dense dusty waist (Woudt et al. 2009). The VISIR last images confirm this since the two lobes appear to be clearly detached from each other in the deconvolved images without any sign of emission close to the equatorial plane (Fig. 7). Chesneau et al. (2008) reported some evidence for a significant departure from spherical symmetries in the latest VLTI data ( $t=145 \mathrm{~d}$ ) from a baseline at more than 60 degree from the major axis. A re-inspection of the data shows that contrary to what was suggested by the authors, the measurements are not affected by any instrumental problem. An aspect ratio as high as 1.3 was probably observed implying that the shaping of the bipolar nebula was already well advanced at this time.

Two questions can be related to these observations. How can this polar ejection be the consequence of a common-envelope phase and what was the time scale of the event? With a different time and spatial scale context, the extreme bipolar planetary nebulae Mz3 and M2-9 can provide enlightening comparisons (Evans 2011). Most of the dust in Mz3 and M2-9 was found in the bipolar lobes (Lykou et al. 2011; Chesneau et al. 2007; Smith \& Gehrz 2005) and recent observations suggest that the time scale of the formation of these nebulae must have been very short, i.e. a few years at most, in a context of a much slower expected evolution of at least several centuries. Regarding M2-9, it is known that the core harbors a long-period binary (Corradi et al. 2011, $P \sim 90 \mathrm{yr}$ ). For these events, the small disks at the core of the systems and the dense lobes favor an intrinsically polar ejection. This hypothesis cannot be ruled out with the formation of the caps of V1280 Sco.

Another scenario implies an intrinsically a-spherical ejection that focuses the mass ejection toward the poles, in which a largescale structured magnetic field may be the shaping key agent. The recent outburst of the recurrent nova T Pyx appeared to be highly bipolar (Chesneau et al. 2011). T Pyx shows a very stable photometric wave that may betray the existence of a magnetic field (Patterson et al. 1998). The presence of a strong magnetic field of about a few MG might have had a non-negligible effect on the eruption, which is also highly suspected for V1500 Cyg stars (Oksanen \& Schaefer 2011).

\section{Conclusion}

We monitored the evolution of the circumstellar material around the classical nova V1280 Sco using high angular resolution infrared observations with the VLT. We resolved it for the first time with a single telescope with the NACO/VLT instrument in the near-infrared and VISIR/VLT in the mid-infrared in 2009, two years after the outburst.

These observations revealed a bipolar-shaped nebula around the nova, with dust present mostly in the lobes of the nebula. The high aspect ratio of the nebula suggests that it is seen at high inclination.

More similar VISIR observations were taken in 2010 and 2011, together with IFU observations with SINFONI/VLT in 2011. This allowed us to observe the expansion of the dusty nebula around V1280 Sco with a rate of $0.39 \pm 0.03$ milliarcsec per day along the major axis of the nebula. Assuming that the dust we observed with these infrared observations expands at the same speed as the low-excitation ejecta detected via spectroscopy, this suggests that the distance to V1280 Sco is at least $1 \mathrm{kpc}$.

Finally, these observations enabled us to study the temporal evolution of a dusty bipolar source. Further yearly monitoring of V1280 Sco will be easier because the nebula is expanding, and will provide an excellent test-case for the study of the envelope dust and gas content.

Acknowledgements. We thank Lowell Tacconi-Garman, Mario van den Ancker and Elena Valenti, among others, for their invaluable assistance with the preparation of these observations. Part of this work was carried out within the framework of the European Associated Laboratory "Astrophysics Poland-France". We thank Pierre Cruzalèbes for his advice on the MARCS synthetic models.

\section{References}

Bernstein, L. S., \& Lynch, D. K. 2009, ApJ, 704, 226

Bode, M. F. 2002, in Classical Nova Explosions, eds. M. Hernanz, \& J. José, AIP Conf. Ser., 637, 497

Chesneau, O. 2011, in Asymmetric Planetary Nebulae 5 Conference

Chesneau, O., Lykou, F., Balick, B., et al. 2007, A\&A, 473, L29 
Chesneau, O., Banerjee, D. P. K., Millour, F., et al. 2008, A\&A, 487, 223 Chesneau, O., Clayton, G. C., Lykou, F., et al. 2009, A\&A, 493, L17 Chesneau, O., Meilland, A., Banerjee, D. P. K., et al. 2011, A\&A, 534, L11

Corradi, R. L. M., Balick, B., \& Santander-García, M. 2011, A\&A, 529, A43

Das, R. K., Banerjee, D. P. K., Ashok, N. M., \& Chesneau, O. 2008, MNRAS, 391,1874

De Marco, O., Passy, J., Moe, M., et al. 2011, MNRAS, 411, 2277

De Marco, O., \& Soker, N. 2011, PASP, 123, 402

Downes, R. A., \& Duerbeck, H. W. 2000, AJ, 120, 2007

Eisenhauer, F., Abuter, R., Bickert, K., et al. 2003, in SPIE Conf. Ser. 4841, eds. M. Iyes, \& A. F. M. Moorwood, 1548

Evans, A. 2011, in Asymmetric Planetary Nebulae 5 Conference

Evans, A., Gehrz, R. D., Geballe, T. R., et al. 2003, AJ, 126, 1981

Evans, A., Tyne, V. H., Smith, O., et al. 2005, MNRAS, 360, 1483

Evans, A., Gehrz, R. D., Woodward, C. E., et al. 2010, MNRAS, 406, L85

Gustafsson, B., Edvardsson, B., Eriksson, K., et al. 2008, A\&A, 486, 951

Harman, D. J., \& O’Brien, T. J. 2003, MNRAS, 344, 1219

Hounsell, R., Bode, M. F., Hick, P. P., et al. 2010, ApJ, 724, 480

Kastner, J. H., Buchanan, C., Sahai, R., Forrest, W. J., \& Sargent, B. A. 2010, AJ, 139, 1993

Kato, M., \& Hachisu, I. 2011, ApJ, 743, 157

Kervella, P., Verhoelst, T., Ridgway, S. T., et al. 2009, A\&A, 504, 115

Kraus, M., Borges Fernandes, M., \& Chesneau, O. 2010, Binaries - Key to Comprehension of the Universein, eds. A. Prša, \& M. Zejda (San Francisco: ASP), 395

Lagadec, E., Verhoelst, T., Mékarnia, D., et al. 2011, MNRAS, 417, 32
Lagage, P. O., Pel, J. W., Authier, M., et al. 2004, The Messenger, 117, 12 Lasker, B. M., Lattanzi, M. G., McLean, B. J., et al. 2008, AJ, 136, 735 Lau, H. H. B., de Marco, O., \& Liu, X. 2011, MNRAS, 410, 1870 Livio, M., Shankar, A., Burkert, A., \& Truran, J. W. 1990, ApJ, 356, 250 Lloyd, H. M., O’Brien, T. J., \& Bode, M. F. 1997, MNRAS, 284, 137 Lyke, J. E., \& Campbell, R. D. 2009, AJ, 138, 1090

Lykou, F., Chesneau, O., Zijlstra, A. A., et al. 2011, A\&A, 527, A105 Lynch, D. K., Rudy, R. J., Russell, R. W., et al. 2009, in A\&AS Meeting Abstracts, 214, 428.08

Meilland, A., Kanaan, S., Borges Fernandes, M., et al. 2010, A\&A, 512, A73

Millour, F., Meilland, A., Chesneau, O., et al. 2011, A\&A, 526, A107

Moraes, M., \& Diaz, M. 2009, AJ, 138, 1541

Naito, H., Mizoguchi, S., Arai, A., et al. 2012, A\&A, 543, A86

Ness, J., Schwarz, G., Woodward, C. E., et al. 2009, The Astronomer's Telegram, 2063, 1

Oksanen, A., \& Schaefer, B. E. 2011, The Astronomer's Telegram, 3782, 1

Patterson, J., Kemp, J., Shambrook, A., et al. 1998, PASP, 110, 380

Porter, J. M., O'Brien, T. J., \& Bode, M. F. 1998, MNRAS, 296, 943

Sadakane, K., Tajitsu, A., Mizoguchi, S., Arai, A., \& Naito, H. 2010, PASJ, 62, L5

Schwarz, G. J., Ness, J.-U., Osborne, J. P., et al. 2011, ApJS, 197, 31

Smith, N., \& Gehrz, R. D. 2005, AJ, 129, 969

Solf, J. 1983, ApJ, 273, 647

Woudt, P. A., Steeghs, D., Karovska, M., et al. 2009, ApJ, 706, 738

Yamaoka, H., Nakamura, Y., Nakano, S., Sakurai, Y., \& Kadota, K. 2007, IAU Circ., 8803, 1 